\title{
Stationary and Dynamic Processes in Process Furnaces
}

\author{
Nikolay D. Demidenko ${ }^{a}$ and Ludmila V. Kulagina*b \\ ${ }^{a} S D T B \ll N a u k a » K S C S B R A S$ \\ 53 Mira, Krasnoyarsk, 660049, Russia \\ ${ }^{b}$ Siberian Federal University \\ 79 Svobodny, Krasnoyarsk, 660041, Russia
}

Received 01.04.2015, received in revised form 18.07.2015, accepted 22.11.2015

In article we propose mathematical models for stationary and dynamic processes of technological furnaces as object with distributed parameters. This mathematical model is based on the laws of conservation of energy, mass and impulse and includes differential equations in private derivatives. Computational investigation of stationary modes of tubular furnaces is conducted. Formulated boundary-value problem for calculation concentration of combustible substance, density, speed, temperature of the flue gas and temperature of the heated raw materials going on division in rectificative column. Results of calculation technological parameters are presented. The proposed method can be used at automation rectificative plant in petroleum-refining and petrochemical industry.

Keywords: mathematical modeling, distributed parameter systems, heat mass exchange, numerical method.

Citation: Demidenko N.D., Kulagina L.V. Stationary and dynamic processes in process furnaces, J. Sib. Fed. Univ. Eng. technol., 2016, 9(1), 6-14. DOI: 10.17516/1999-494X-2016-9-1-6-14.

\section{Стационарные и динамические процессы \\ в технологических печах}

\author{
Н.Д. Демиденко ${ }^{\mathrm{a}}$, Л.В. Кулагина ${ }^{\mathbf{\sigma}}$ \\ ${ }^{a}$ СКТБ «Наука» КНЦ СО РАН \\ Россия, 660049, Красноярск, пр. Мира, 53 \\ ${ }^{6}$ Сибирский федеральный университет \\ Россия, 660041, Красноярск, пр. Свободный, 79
}

В статье предложены математические модели для стационарных и динамических процессов технологических печей как объектов с распределёнными параметрами. Эти математические

(C) Siberian Federal University. All rights reserved

* Corresponding author E-mail address: klvation@gmail.com 
модели получены на основе законов сохранения энергии, массы и импульса и включают в себя дифференциальные уравнения в частных производных. Проведено численное исследование стационарных режимов трубчатых печей. Для этого сформулирована краевая задача для расчета кониентрачии горючего вещества, плотности, скорости, температуры дымовых газов и температуры нагреваемого сырья, идущего на разделение в ректификачионную колонну. Представлены результаты расчета технологических параметров. Предложенныйметодможет бытьиспользованприавтоматизацииректификаиионныхустановоквнефтеперерабатывающей и нефтехимической промышленности.

Ключевые слова: математическое моделирование, системы с распределёнными параметрами, тепломассообмен, численный метод.

Для высокотемпературного нагрева нефти и нефтепродуктов в процессе их переработки в ректификационных установках применяют трубчатые печи. Печи работают следующим образом [1]. Мазут и газ сжигаются в горелках, расположенных в камере радиации. Продукты сгорания из камеры радиации поступают в камеру конвекции, затем направляются в газосборник и по дымовой трубе уходят в атмосферу. В камере конвекции расположены конвекционные трубы, воспринимающие тепло при соприкосновении дымовых газов с поверхностью нагрева путем конвекции. Нагреваемый продукт в печи последовательно проходит через конвекционные и радиантные трубы, поглощая тепло. Радиантная поверхность воспринимает большую часть тепла, выделяемого при сгорании топлива (рис. 1).

Горение жидкого топлива всегда протекает в паровой фазе, причем испарение обеспечивается подводом тепла из зоны горения паров. Топливо всегда сжигается в распыленном виде. Крайним случаем горения капель является диффузионное горение, когда скорость сгорания паров очень велика по отношению к скорости диффузии паров и окислителя в зоне горения, толщина которой становится исчезающе малой. К такому режиму может приближаться горение сравнительно круглых капель. Диффузионная теория горения развита Г.А. Варшавским [2].

\section{Математические модели теплофизических процессов}

В математических методах анализа процесса горения у разных авторов имеются некоторые расхождения, но для стационарного сферического горения используется единый подход. В целях упрощения анализ проводится при следующих предположениях:

1. Жидкая капля имеет сферическую форму.

2. Влиянием конвекции пренебрегают, пламя рассматривают как сферическую поверхность, концентрическую с каплей.

3. Пламя считают разновидностью диффузионного пламени, которое образуется в результате реакции между парами горючего и воздухом, которые реагируют в стехиометрическом соотношении.

4. Рассматривают стационарное состояние при постоянном диаметре капли, хотя реально диаметр жидкой капли уменьшается по мере горения, однако это изменение происходит медленно по сравнению с изменением скорости диффузии и прочими факторами.

5. Температура капли одинакова по всему объему.

6. Давление в течение всего процесса горения считается постоянным.

7. Влияние излучения рассматривают отдельно [36]. 


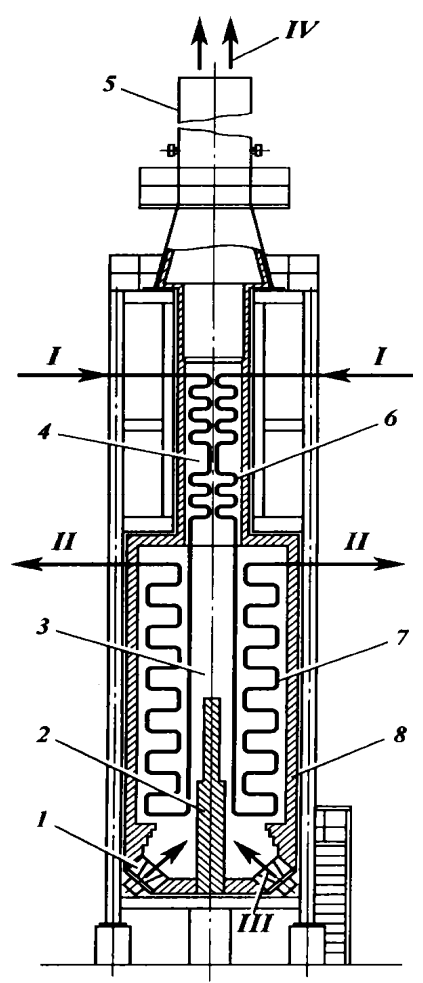

Рис. 1. Схема трубчатой печи: 1 - горелка; 2 - настильная стенка; 3 - камера радиации (топочная камера); 4 - камера конвекции; 5 - дымовая труба; 6 - змеевик конвекционных труб; 7 - змеевик радиантных труб; 8 - футеровка. Потоки: I - вход сырья; II - выход сырья; III - топливо и воздух; IV - дымовые газы

Исходя из законов механики сплошных сред, можно получить следующие уравнения нестационарного горения:

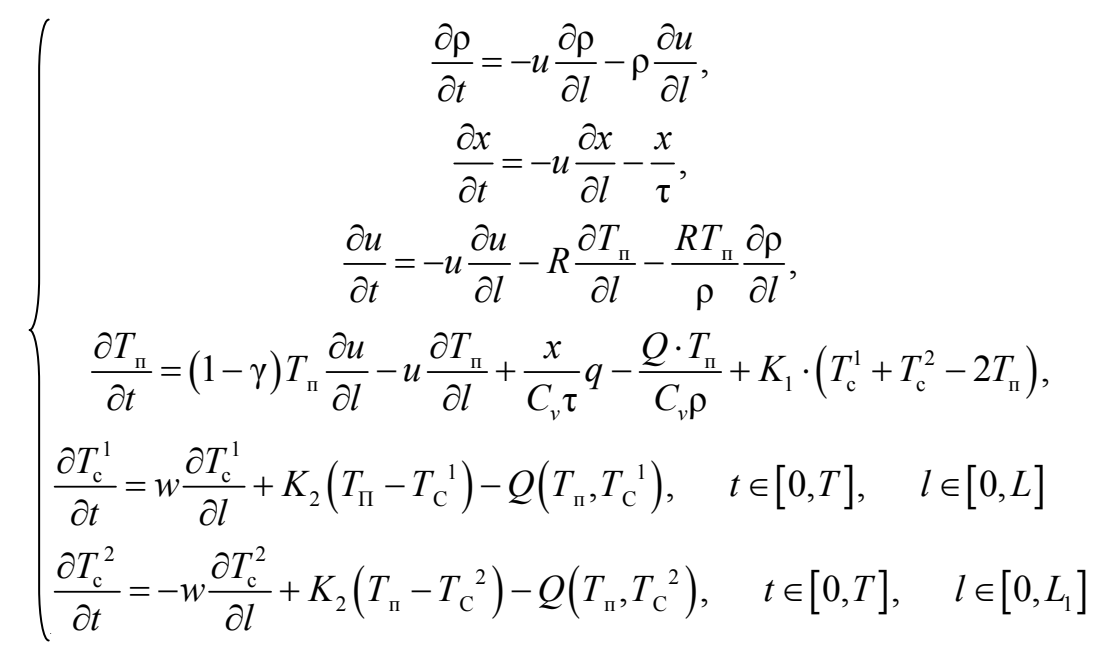

где $\rho$ - массовая плотность смеси; $t$ - время; $u$ - скорость движения смеси, $l$ - пространственная координата; $x$ - концентрация горючего вещества в смеси $(0 \leq x \leq 1) ; \tau-$ время сгорания; $R$ - газовая постоянная; $q$ - теплота сгорания топлива; $T_{n}$ - температура дымовых газов; $Q(T)$ потери тепла на излучение; $(\gamma=1 \div 1,4)$ - показатель адиабаты; $C_{v}$ - теплоемкость газов при 
постоянном объеме; $w$ - скорость сырья (нефтепродукта в радиантных трубопроводах печи); $K_{1}$ - коэффициент теплопередачи для рабочего потока, $T_{C}{ }^{1}, T_{C}{ }^{2}$ - температура нисходящего и восходящего потоков нагреваемого сырья соответственно; $K_{2}$ - коэффициент теплопередачи для стенки печи; $L$ - длина печи.

$$
\begin{gathered}
\frac{\partial \rho}{\partial t}=-u \cdot \frac{\partial \rho}{\partial l}-c \cdot \frac{\partial u}{\partial l}, \\
\frac{\partial x}{\partial t}=-u \cdot \frac{\partial x}{\partial l}-\frac{x}{\varphi}, \\
\frac{\partial u}{\partial t}=-u \cdot \frac{\partial u}{\partial l}-R \cdot \frac{\partial T_{n}}{\partial l}-\frac{R T_{n}}{c} \cdot \frac{\partial \rho}{\partial l}, \\
\frac{\partial T_{n}}{\partial t}=(1-u) \cdot T_{n} \cdot \frac{\partial u}{\partial l}-u \cdot \frac{\partial T_{n}}{\partial l}+\frac{x}{C_{v} \cdot \varphi} \cdot q-\frac{Q \cdot T_{n}}{C_{v} \cdot c}+K_{1} \cdot\left(T_{c}^{1}+T_{c}^{2}-2 T_{n}\right), \\
\frac{\partial T_{c}^{1}}{\partial t}=-w \cdot \frac{\partial T_{c}^{1}}{\partial l}+K_{2} \cdot\left(T_{n}-T_{c}^{1}\right)-Q\left(T_{n}, T_{c}^{1}\right), \\
\frac{\partial T_{c}^{2}}{\partial t}=-w \cdot \frac{\partial T_{c}^{2}}{\partial l}+K_{2} \cdot\left(T_{n}-T_{c}^{2}\right)-Q\left(T_{n}, T_{c}^{2}\right)=0 .
\end{gathered}
$$

Здесь $x(l, t)$ - концентрация горючего вещества, $\rho(l, t), u(l, t), T_{n}(l, t)-$ плотность, скорость и температура дымовых газов; $T_{c}(l, t), T_{c}{ }_{c}(l, t)$ - температура нисходящего и восходящего потоков нагреваемого сырья; $t, l$ - временная и пространственная координаты.

Начальные условия

$$
x(l, 0)=\varphi_{1}(l), \quad u(l, 0)=\varphi_{2}(l), \quad T(l, 0)=\varphi_{3}(l), \quad T_{c}(l, 0)=\varphi_{4}(l) .
$$

Граничные условия

$$
x(0, t)=\varphi_{1}(t), \quad u(0, t)=\varphi_{2}(t), \quad T(0, t)=\varphi_{3}(t), \quad T_{c}(0, t)=\varphi_{4}(t) .
$$

Стационарную модель, которая следует из (1), исследуем более подробно:

$$
\begin{gathered}
-u \cdot \frac{\partial \rho}{\partial l}-\rho \cdot \frac{\partial u}{\partial l}=0 \\
-u \cdot \frac{\partial x}{\partial l}-\frac{x}{\tau}=0 \\
-u \cdot \frac{\partial u}{\partial l}-R \cdot \frac{\partial T_{n}}{\partial l}-\frac{R T_{n}}{\rho} \cdot \frac{\partial \rho}{\partial l}=0 \\
(1-\gamma) \cdot T_{\text {п }} \cdot \frac{\partial u}{\partial l}-u \cdot \frac{\partial T_{\mathrm{n}}}{\partial l}+\frac{x}{C_{v} \cdot \tau} \cdot q-\frac{Q \cdot T_{\mathrm{n}}}{C_{v} \cdot \rho}+K_{1} \cdot\left(T_{c}^{1}+T_{c}^{2}-2 T_{\mathrm{n}}\right)=0, \\
w \cdot \frac{\partial T_{\mathrm{c}}^{1}}{\partial l}+K_{2} \cdot\left(T_{\mathrm{n}}-T_{\mathrm{c}}^{1}\right)-Q\left(T_{\mathrm{n}}, T_{\mathrm{c}}^{1}\right)=0, \\
-w \cdot \frac{\partial T_{\mathrm{c}}^{2}}{\partial l}+K_{2} \cdot\left(T_{\mathrm{n}}-T_{c}^{2}\right)-Q\left(T_{\mathrm{n}}, T_{\mathrm{c}}^{2}\right)=0 . \\
-9-
\end{gathered}
$$


Второе, пятое и шестое уравнения из (5) содержат пространственные производные только одной неизвестной функции. Приведем первое, третье и четвертое уравнения к виду, содержащему производную только от одной искомой функции $\mathrm{d} \rho / \mathrm{d} l \mathrm{~d} u / \mathrm{d} l$ и $\mathrm{d} T_{\text {п }} / \mathrm{d} l$. Для этого применим теорию о неявных функциях к системе.

$$
\begin{gathered}
u \cdot \frac{\partial \rho}{\partial l}+\rho \cdot \frac{\partial u}{\partial l}=0, \\
u \cdot \frac{\partial u}{\partial l}+R \cdot \frac{\partial T_{\mathrm{n}}}{\partial l}+\frac{R T_{\mathrm{n}}}{\rho} \cdot \frac{\partial \rho}{\partial l}=0, \\
(1-\gamma) \cdot T_{\text {п }} \cdot \frac{\partial u}{\partial l}-u \cdot \frac{\partial T_{\mathrm{I}}}{\partial l}=-\frac{x}{C_{v} \cdot \tau} \cdot q+\frac{Q \cdot T_{\mathrm{n}}}{C_{v} \cdot \rho}-K_{1} \cdot\left(T_{c}^{1}+T_{c}^{2}-2 T_{\text {п }}\right)=w,
\end{gathered}
$$

В этом случае

$$
\frac{\partial \rho}{\partial l}=\frac{\Delta_{1}}{\Delta}, \quad \frac{\partial u}{\partial l}=\frac{\Delta_{2}}{\Delta}, \quad \frac{\partial T_{\Pi}}{\partial l}=\frac{\Delta_{3}}{\Delta},
$$

где

$$
\begin{aligned}
\Delta & =\left|\begin{array}{ccc}
u & \rho & 0 \\
\frac{R T_{\text {п }}}{\rho} & u & R \\
0 & (1-\gamma) T_{\text {п }} & -u
\end{array}\right|=-u^{3}+u \rho \frac{R T_{\text {п }}}{\rho}-u R(1-\gamma) T_{\text {п }}=-u^{3}+\gamma R u T_{\text {п }}=u\left(\gamma R T_{\text {п }}-u^{2}\right) \\
\Delta_{1} & =\left|\begin{array}{ccc}
u & \rho & 0 \\
0 & u & R \\
\tau & (1-\gamma) T_{\text {п }} & -u
\end{array}\right|=R \rho \tau, \Delta_{2}=\left|\begin{array}{ccc}
u & \rho & 0 \\
\frac{R T_{\text {п }}}{\rho} & u & R \\
0 & \tau & -u
\end{array}\right|=R \rho \tau, \\
T_{\text {п }} & =\left|\begin{array}{ccc}
u & \rho & 0 \\
R T_{\text {п }} & u & R \\
\rho & & \\
0 & (1-\gamma) T_{\text {п }} & \tau
\end{array}\right|=u^{2} \tau .
\end{aligned}
$$

Таким образом, будем иметь:

$$
\begin{aligned}
& \frac{\partial \rho}{\partial l}=\frac{\Delta_{1}}{\Delta}=\frac{R c w}{u\left(\gamma R T_{\text {п }}-u^{2}\right)}, \\
& \frac{\partial u}{\partial l}=\frac{\Delta_{2}}{\Delta}=-\frac{R u w}{u\left(\gamma R T_{\text {п }}-u^{2}\right)}=\frac{R w}{\gamma R T_{\text {п }}-u^{2}}, \\
& \frac{\partial T_{\text {п }}}{\partial l}=\frac{\Delta_{3}}{\Delta}=\frac{u^{2} w}{u\left(\gamma R T_{\text {п }}-u^{2}\right)}=\frac{u w}{\gamma R T_{\text {п }}-u^{2}}
\end{aligned}
$$

Тем самым система (4) приведена к следующему виду: 


$$
\begin{gathered}
\frac{\Delta \rho}{\Delta l}=\frac{R \rho\left(-\frac{x}{C_{v} \tau} q+\frac{Q T_{\mathrm{n}}}{C_{\nu} \rho}-K_{1}\left(T_{\mathrm{c}}^{1}+T_{\mathrm{c}}^{2}-2 T_{\text {п }}\right)\right)}{u\left(\gamma R T_{\mathrm{n}}-u^{2}\right)}, \\
\frac{\Delta T_{\mathrm{n}}}{\Delta l}=\frac{u\left(-\frac{x}{C_{v} \tau} q+\frac{Q T_{\mathrm{n}}}{C_{v} \rho}-K_{1}\left(T_{\mathrm{c}}^{1}+T_{\mathrm{c}}^{2}-2 T_{\text {п }}\right)\right)}{\gamma R T_{\text {п }}-u^{2}} \\
\frac{\Delta u}{\Delta l}=\frac{-R\left(-\frac{x}{C_{v} \tau} q+\frac{Q T_{\text {п }}}{C_{v} \rho}-K_{1}\left(T_{\mathrm{c}}^{1}+T_{\mathrm{c}}^{2}-2 T_{\text {п }}\right)\right)}{\gamma R T_{\text {п }}-u^{2}} \\
\frac{\partial T_{\mathrm{c}}^{1}}{\partial l}=\frac{K_{2}}{w}\left(T_{\text {п }}-T_{\mathrm{c}}^{1}\right)-\frac{1}{w} Q\left(T_{\mathrm{n}}, T_{\mathrm{c}}^{1}\right), \\
\frac{\partial T_{\mathrm{c}}^{2}}{\partial l}=\frac{K_{2}}{w}\left(T_{\mathrm{n}}-T_{\mathrm{c}}^{2}\right)-\frac{1}{w} Q\left(T_{\mathrm{n}}, T_{\mathrm{c}}^{2}\right) . \\
\rho(0)=\alpha_{1}, \quad x(0)=\alpha_{2}, \quad u(0)=\alpha_{3}, \\
T_{\text {п }}(0)=\alpha_{4}, \quad T_{\mathrm{c}}^{1}(0)=\alpha_{5}, \quad T_{\mathrm{c}}^{2}(0)=T_{\mathrm{c}}^{1}(0) .
\end{gathered}
$$

\section{Результаты численных исследований стационарных режимов}

На рис. 2-5 отражены результаты расчетов стационарных режимов при различных начальных условиях для плотности $\rho(l)$, концентрации $x(l)$, скорости $u(l)$ и температуры дымовых газов $T_{\text {п }}(l)$. При этом за начальные условия приняты $\rho(0)=720$ кг $/ \mathrm{M}^{3}, x(0)=0,47, u(0)=5 \mathrm{м} / \mathrm{c}$, $T_{\text {п }}(0)=450{ }^{\circ} \mathrm{C}, T_{\mathrm{c}}^{1}(L)=270{ }^{\circ} \mathrm{C}$. Затем начальные данные изменялись с шагом $\pm 5 \%$ при постоянных значениях остальных параметров.

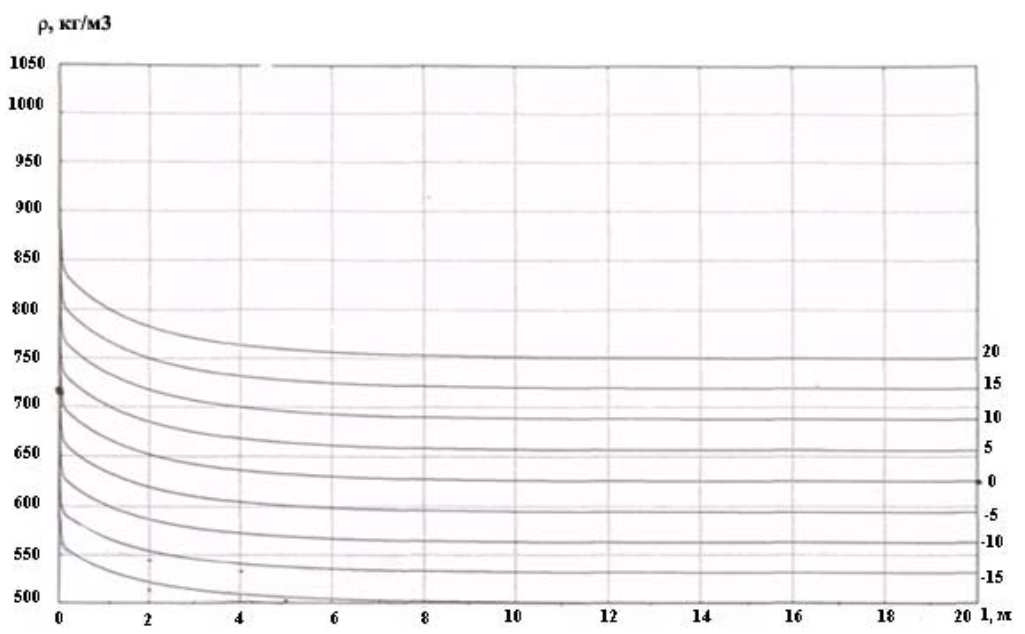

Рис. 2. Распределение плотности дымовых газов по длине объекта 


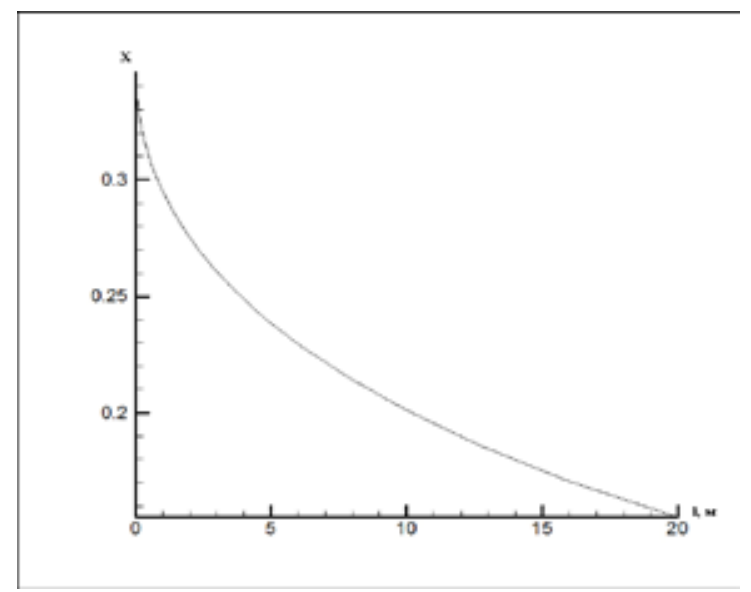

Рис. 3. Изменения концентрации горючего вещества

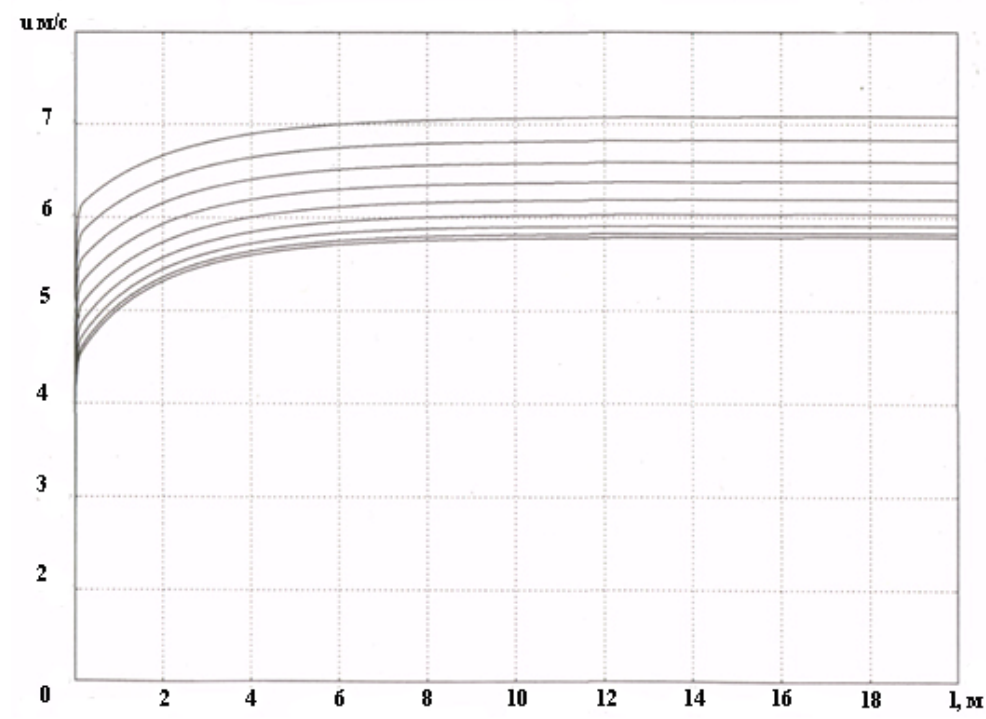

Рис. 4. Изменения скорости дымовых газов

Полученные результаты дают возможность сделать следующие заключения:

- увеличение плотности потока на выходе из печи происходит в силу снижения скорости потока газа и продуктов сгорания. На рис. 3 отражено выгорание топлива. При детальном рассмотрении процесса с помощью двумерных зависимостей [7] наблюдается оптимум в области температур вблизи $450{ }^{\circ} \mathrm{C}$. Увеличение плотности потока выше оптимального приводит к увеличению недожога, снижению КПД процесса и увеличению количества вредных выбросов в окружающую среду. Снижение плотности характеризует недогрев сырья и, соответственно, неоптимальный режим работы технологической установки в целом;

- выявлен эффект перераспределения теплообмена между потоком горячих газов и сырьем по длине печи (рис. 5), что может оказать влияние на качество получаемого про- 


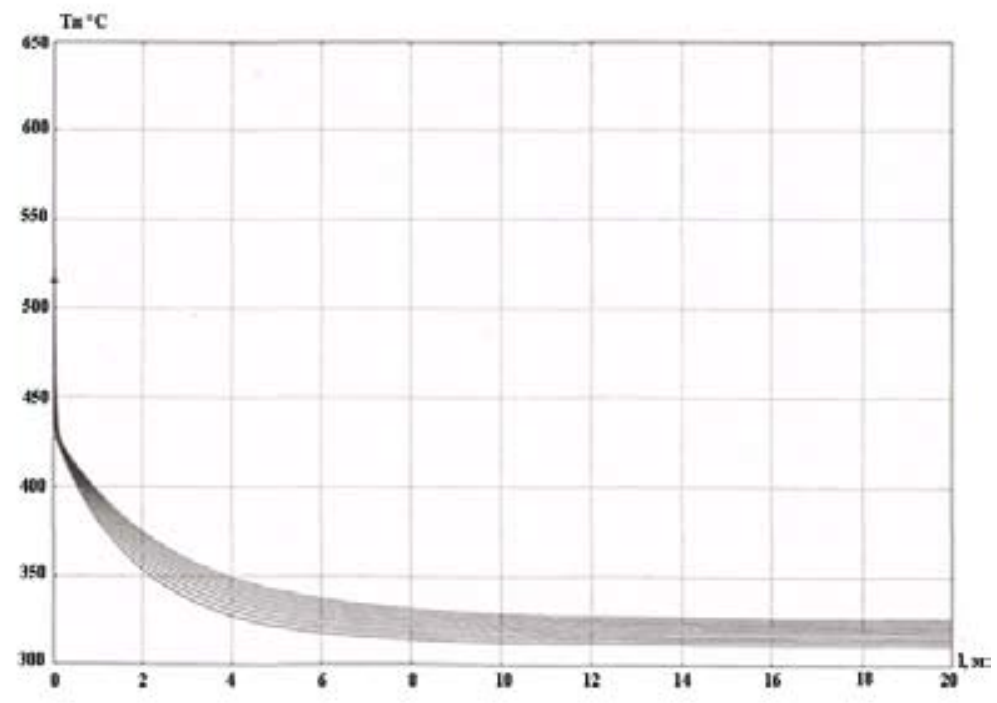

Рис. 5. Изменения температуры дымовых газов

дукта, следовательно, изменяя температуру потока по длине печи или задавая режим работы, можно получать продукт заданного наперед качества;

- управляя скоростью движения потока (рис. 4), имеется возможность регулировать плотность и, как следствие, величину недожога и, значит, количество выбросов в окружающую среду на выходе из печи.

Сопоставление полученных результатов со значениями управляющих параметров реального производства АНПЗ [7] показало хорошее соответствие (отличие составляет 1-5 \%).

\section{Вывод}

Предлагаемый метод анализа стационарных режимов технологических печей позволяет еще на стации проектирования ректификационных установок обеспечить высокое качество разделения многокомпонентных смесей в нефтепереработке и нефтехимии.

\section{Список литературы}

[1] Скобло А.И., Молоканов Ю.К., Владимиров А.И., Щелкунов В.А. Процессы и апnараты нефтегазопереработки и нефтехимии. М.: ООО «Недра-Бизнесцентр», 2000, 677 с. [Skoblo A.I., Molokanov Iu.K., Vladimirov A.I., Shchelkunov V.A. Processes and devices of oil \& gas and petrochemical, Moscow, Nedra-Biznestsentr, 2000, 677 p. (in Russian)]

[2] Варшавский Г.А. Горение капли жидкого топлива (диффузионная теория). Бюро новой техники НКАП, 1945, 6, 87-106 [Varshavskii G.A. Burning droplets of liquid fuel (diffusion theory), Biuro novoi tekhniki NKAP, 1945, 6, 87-106 (in Russian)]

[3] Демиденко Н.Д. Моделирование статических и динамических режимов в трубчатых печах. Вестник ТомГУ. Управление, вычислительная техника и информатика, 2012, 3(20), 13-21 [Demidenko N.D. Modeling of static and dynamic modes in tube furnaces, Tomsk State University Journal of Control and Computer Science, 2012, 3(20), 13-21 (in Russian)] 
[4] Демиденко Н.Д., Кулагин В.А., Шокин Ю.И. Моделирование и вычислительные технологии распределенных систем. Новосибирск: Наука, 2012, 424 с. [Demidenko N.D., Kulagin V.A., Syokin Iu.I. Modeling and computational technology of the distributed systems, Novosibirsk, Nauka, 2012, 424 p. (in Russian)]

[5] Демиденко Н.Д., Кулагин В.А., Шокин Ю.И., Ли Ф.-Ч. Тепломассообмен и суперкавитация. Новосибирск: Наука, 2015, 436 с. [Demidenko N.D., Kulagin V.A., Syokin Iu.I., Li F.-Ch. Heat and mass transfer and supercavitation, Novosibirsk, Nauka, 2015, 436 p. (in Russian)]

[6] Демиденко Н.Д., Кулагина Л.В. Моделирование и оптимизация технических систем с распределенными параметрами. Красноярск: ИПЦ КГТУ, 2006, 210 с. [Demidenko N.D., Kulagina L.V. Simulation and optimization of technical systems with distributed parameters, Krasnoyarsk, 2006, 210 p. (in Russian)]

[7] Демиденко Н.Д., Кулагина Л.В. Численное исследование течений в трубчатых теплообменниках. Журнал СФУ. Техника и технологии, 2013, 6(5), 506-514 [Demidenko N.D., Kulagina L.V. Computational Investigation of Flows in Tube Heat Exchangers, J. Sib. Fed. Univ. Eng. technol., 2013, 6(5), 506-514 (in Russian)] 